\title{
CHILENIZANDO EL HABITAR. CAMBIOS E INCORPORACIONES EN EL HABITAR DOMÉSTICO DE LOS AYMARAS URBANOS EN LA CIUDAD DE ARICA
}

\author{
CHILENIZANDO THE INHABITING, CHANGES AND ADDITIONS \\ ON THE DOMESTIC SPACES OF THE URBAN AYMARAS IN ARICA
}

\author{
Diego González Carrasco*
}

\begin{abstract}
Este artículo busca ayudar a entender la realidad construida de las viviendas urbanas de las familias aymaras residentes en la ciudad de Arica, en el extremo norte de Chile, luego de los procesos migratorios desarrollados en las últimas décadas. Nos enfocamos en uno de los resultados de un estudio más amplio, centrado en las familias aymaras beneficiarias de viviendas sociales entregadas por el Estado durante las décadas de 1990 y 2000. Describiendo las características de las viviendas tradicionales y aquellas de las unidades habitacionales básicas entregadas por el Estado, podemos observar que estas últimas proponen un programa de espacios predefinido basado en una concepción homogénea del habitar occidental y que junto con la necesidad de asimilarse a la sociedad urbana mayoritaria, han provocado la incorporación y valorización de nuevos espacios construidos que se manifiestan junto con un mobiliario específico, ajenos a las estructuras del habitar tradicional de las viviendas Aymaras.
\end{abstract}

Palabras claves: Vivienda aymara, aymaras urbanos, arquitectura vernácula.

This article look to help. in the understanding of the constructed reality of the urban housing of the Aymara families living in the city of Arica, in the northern Chile, after the migratory processes produced in the last decades. We focus on one of the results of a larger study, focused on Aymara families beneficiaries of social housing provided by the state during the decades of 1990 and 2000. Describing the characteristics of traditional dwellings and those of the basic housing units provided by the state, we can observe that these last ones propose a program of pre defined spaces based on a homogeneous conception of the western inhabiting and that together with the need to assimilation to the majority of the urban society, have led to the incorporation and valorization of new built spaces that are manifested together with a specific furniture, unrelated to the structures of the traditional inhabiting of the Aymaras dwellings.

Key words: Aymara housing, Urban Aymaras, Vernacular Architecture.

\section{Introducción}

Una de las principales consecuencias que han traído los procesos de modernización, especialmente en los países del tercer mundo, lo constituye la urbanización de la población ocurrida con fuerza a partir de la segunda mitad del siglo XX. El traslado masivo a los centros urbanos provoca un "despoblamiento" de las localidades rurales y el incremento explosivo del número de residentes en las ciudades de los países menos desarrollados. Este fenómeno afecta también a los pueblos indígenas. En Chile, de acuerdo con los datos del Censo del 2002, el $63,4 \%$ de la población indígena chilena vivía en zonas urbanas (INE 2003). La salida de sus comunidades de origen, ubicadas en los sectores rurales, no solo involucra el "viaje" a un nuevo espacio de ocupación citadino, sino la incorporación "forzada" de nuevos modelos de habitar que vienen dados por medio de las decisiones globalizadoras del hacer constructivo presente en las ciudades; además de una presión, directa e indirecta, de una sociedad "chilena" que los ve como "otros".

El término chilenización, desde un punto de vista histórico, se refiere a la campaña emprendida una vez finalizada la Guerra del Pacífico (18791883), que buscaba crear en los territorios anexados, luego de la victoria militar, un vínculo ideológico entre la población local (no chilena) y el Estado chileno, nuevo dueño del territorio (Tudela 1993). Esta se vio caracterizada por la acción sistemática del Estado, por ejemplo, reforzando su presencia mediante la instalación de retenes policiales y escuelas en los sectores rurales y con campañas de grupos civiles, las denominadas "Ligas patrióticas" (González, 2004), que con actos violentos terminaron por crear un clima de inseguridad y rechazo de la población peruana aún residente, la que terminó en su mayoría por migrar de la zona de Arica. En este trabajo proponemos retomar el

* Pontificia Universidad Católica de Chile, Escuela de Arquitectura, Santiago, Chile. Correo Electrónico: dngonzal@uc.cl 
término, esta vez otorgándole un sentido actual, respecto de cómo producto de la migración la población aymara en Arica se vio influenciada por la población "chilena" y terminó por adaptar sus modos de habitar, incorporando, como veremos, ciertos aspectos de la vivienda occidental.

\section{La investigación}

El siguiente artículo busca exponer uno de los temas resultantes de la investigación doctoral titulada Inhabiting in the City: the Aymara People in Arica, Chile. Social and Cultural Factors in Government Housing Programmes an Interdisciplinary Study llevada a cabo en el marco de la obtención del título de $\mathrm{PhD}$ en Arquitectura en la Escuela de Arquitectura de la Universidad de Sheffield en Reino Unido.

Una de las principales consecuencias que han tenido los procesos de modernización, especialmente en los países del tercer mundo, lo constituye la urbanización de la población ocurrida con fuerza a partir de la segunda mitad del siglo XX. El traslado masivo a los centros urbanos provoca un "despoblamiento" de las localidades rurales y el incremento explosivo del número de residentes en las ciudades de los países menos desarrollados. Este fenómeno afecta también a los pueblos indígenas. De acuerdo con los datos del Censo del 2002, el 63,4\% de la población indígena chilena vivía en zonas urbanas (INE, 2005). La salida de sus comunidades de origen, ubicadas en los sectores rurales, no solo involucra el "viaje" a un nuevo espacio de ocupación citadino, sino la incorporación "forzada" de nuevos modelos de habitar que vienen dados por las decisiones globalizadoras del hacer constructivo presente en las ciudades, además de una presión directa e indirecta de una sociedad "chilena" que los ve como "otros". Una manera de poder entender los efectos del traslado a las áreas urbanas de la población rural en general y de los grupos indígenas en particular, desde el ámbito de la arquitectura, es la revisión de los espacios construidos resultantes de este proceso. La arquitectura vernácula -autóctona, tradicional, popular- ofrece la posibilidad de abordar no solo los aspectos que han sido desarrollados por investigadores y estudiosos de las ciencias sociales, sino también respecto de la forma en que se adaptan a la vida en la ciudad, la materialidad y métodos constructivos que responden de manera correcta a realidades geográficas, climáticas y culturales específicas a sus áreas de origen.
Este trabajo de investigación consistió en analizar la situación actual de familias aymaras que habitan en viviendas sociales entregadas por el Estado chileno en la ciudad de Arica. Desde ese punto específico de partida se buscó contribuir al entendimiento de la realidad de este grupo indígena respecto de su habitar, intentando poner en valor el análisis de las arquitecturas vernáculas como una herramienta a ser utilizada en el desarrollo de respuestas en áreas donde varias culturas coexisten como es el caso de esta ciudad en el norte de Chile.

La hipótesis desde la que se dio inicio al trabajo fue de que a pesar de los cambios causados por la migración a las ciudades costeras, aún era posible encontrar características del habitar tradicional en las viviendas de familias aymaras beneficiarias de los programas de vivienda social del Estado chileno durante las décadas de 1990 y 2000.

Para realizar este estudio de la realidad actual del habitar en viviendas sociales ampliadas mediante la autoconstrucción fue necesario indagar primero acerca del habitar tradicional aymara, de manera de caracterizar las tipologías y significaciones simbólicas que se esperaban trasladadas al nuevo espacio de residencia urbana. Así también se realizó un trabajo bibliográfico que buscó contextualizar la solución habitacional de la que fueron beneficiarios las familias aymaras estudiadas en un marco más amplio del desarrollo histórico de políticas de vivienda a nivel nacional. Mediante el estudio de 12 casos seleccionados se llevó a cabo una investigación que incluyó la aplicación de entrevistas semiestructuradas, levantamiento planimétrico de viviendas urbanas y tradicionales, además de un registro fotográfico.

Entre los resultados que arrojó el análisis de la información recopilada se registró una constante asimilación de ciertos espacios de la vivienda occidental, particularmente el "living" o "sala de estar", que fueron analizados en sus realidades tanto espacial/construida y en términos de la utilización de ciertos tipos de mobiliario. Este hecho se revisó como manifestación inmueble y mueble de una cierta necesidad de aceptación y asimilación frente a la sociedad chilena no indígena que mayoritariamente habita la ciudad.

\section{La vivienda tradicional}

Como se mencionó en líneas anteriores, para entender el alcance de la nueva realidad de habitación 
urbana en viviendas sociales de las familias aymaras en Arica, tanto respecto de la mantención o traslado de elementos del habitar tradicional como de los cambios o incorporaciones en la manera de construir y habitar el espacio doméstico, se hace necesario conocer primero las características fundamentales y comunes que poseen las viviendas aymaras en los espacios geográficos de origen.

Basándonos en los cronistas españoles, la vivienda andina prehispánica se componía de uno o dos volúmenes rectangulares, con muros de piedras $\mathrm{y}$ una techumbre a dos aguas construida en maderas y cubierta de una mezcla de paja y barro (Canals 1959). Sin embargo, podemos encontrar relatos de viviendas con planta circular en zonas más frías y en la Sierra (Muñoz 2013). Una de las características principales de los asentamientos andinos era la ocupación a modo de archipiélagos poblacionales (González, Gundermann e Hidalgo 2014), donde las mayores concentraciones de viviendas estaban dadas por núcleos familiares extendidos y no en una lógica de poblado como se puede entender bajo la lógica occidental. Relevante es también mencionar que la ocupación territorial se daba en todos los pisos ecológicos (costa, valles bajos, valles altos y altiplano), previo a la llegada de los españoles. Es precisamente con la llegada de los colonizadores y particularmente después de 1570 cuando el virrey Francisco de Toledo dicta un grupo de leyes que significarán un cambio de relevancia en la manera de vivir de las poblaciones andinas (Muñoz 2013). Las leyes toledanas, en este ámbito, tendrán como objetivo principal el agrupar la dispersa población indígena y concentrarla en nuevos centros poblados españoles. Estos pueblos recogerán buena parte de las lógicas de fundación de ciudades, utilizando la grilla ortogonal, conformando un damero, con una plaza central franqueada por una iglesia, particularmente relevante en el proceso de evangelización. El advenimiento de esta nueva forma de habitar tendrá cambios importantes en el modo de vida de las poblaciones andinas, sin embargo, no podrá cambiar el hecho de que la economía agrícola y ganadera (en valles y en las zonas altas, respectivamente) seguirá siendo la principal fuente de subsistencia, provocando que las familias mantengan las viviendas en los territorios de cultivo o de pastoreo, sumando una nueva vivienda en los poblados españoles, generándose progresivamente una situación de multiplicidad de viviendas, que se mantendrá hasta nuestros días.

En el caso del altiplano de Arica, donde se realizó el trabajo en terreno para la investigación, pudimos constatar que se reconoce una vivienda principal o uta como la más importante y donde pasan la mayor parte del tiempo en los lugares de origen, la realidad geográfica de estos espacios y el hecho de ser la ganadería la fuente principal de subsistencia, los obliga a desplazarse en busca de áreas de pastoreo por el territorio (Figura 1). Debido a esta labor, permanecen alejados de la vivienda principal por incluso varios días, alojando en viviendas temporales o paskanas. Estos refugios de precaria construcción se encuentran dispuestos en las zonas de pastoreo para su uso estacional. Así también, en el pueblo principal o marka mantienen otra vivienda, la que normalmente se utiliza durante los días de celebraciones religiosas. A esta realidad tradicional de multiplicidad de espacios de habitación o refugio debemos sumar hoy la vivienda urbana, la que en todos los casos estudiados se transformó en la vivienda principal; es decir, aquella donde pasan la mayor parte del año debido a la migración del núcleo familiar casi en su totalidad a la ciudad.

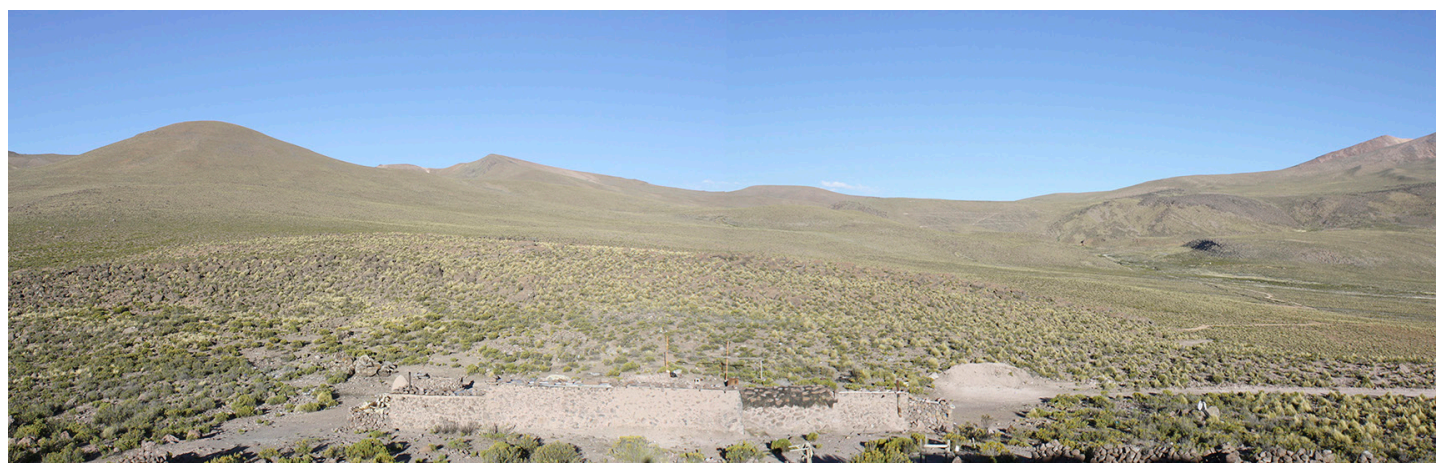

Figura 1. Vivienda tradicional Aymara uta en Itiza, Sector altiplano norte. 
Solo los adultos mayores fueron nombrados como habitantes frecuentes de las utas, generalmente son las abuelas o abuelos los que terminan haciéndose cargo de algunos animales (llamas o alpacas) que la familia mantiene en la estancia.

La uta consiste en varias piezas de planta rectangular, construidas como módulos independientes y con programas diferenciados claramente: cocina, dormitorios y despensa. El principal lugar de la vivienda es la cocina, espacio en el que se agrupa la mayoría de las actividades interiores diarias y de encuentro social. Desde el punto de vista de sus características constructivas, el sistema de techos es de dos aguas y la puerta de entrada está ubicada normalmente hacia el este, para recibir los primeros rayos del sol en las mañanas (Van Kessel 1996). Tradicionalmente carece de ventanas y si estas aparecen son de un tamaño reducido para protegerse mejor de las extremas temperaturas que pueden registrarse en la zona altiplánica durante las noches, que pueden llegar a registrar temperaturas bajo los cero grados (Aceituno 1996).

La materialidad de cada uno de estos módulos que componen la vivienda se explica por los materiales que son posibles de encontrar en la zona. Así, el principal elemento de construcción utilizado será el adobe, viéndose también el uso de piedra particularmente en los sectores de valles altos. Los muros se levantan sobre una base de piedras que sirve de fundación, mientras que encontraremos vigas de madera que sostienen el sistema de techumbre. El techo se construye mediante el uso de palos de madera que se amarran con cueros húmedos, los que al secar se encogen y afirman las piezas en su lugar. Respecto del envigado, se colocan planchas delgadas de adobe, las que luego se cubren con paja suelta para finalizar con una última capa exterior compuesta de atados de paja de mayor tamaño (Solc 1975). Estos módulos construidos se agrupan normalmente alrededor de un patio, espacio exterior que juega un rol fundamental en la manera de habitar la vivienda, siendo un espacio exterior doméstico donde se realizan diversas labores (González y Carrasco 2016).

En la manera en que se desarrolla el habitar doméstico en la vivienda aymara tradicional, el mobiliario dispuesto en ella parece responder únicamente a una lógica de uso, sin observarse una necesidad de disponer de nada más que lo estrictamente necesario para realizar las tareas domésticas diarias. Por ejemplo, para guardar utensilios o víveres se observan cajas de madera o sacos, no viéndose comúnmente el uso de armarios o cajoneras. En el espacio interior más relevante, la cocina, el mobiliario tradicional se compone de un asiento corrido construido de piedra o adobe que recorre las paredes de la habitación (Figura 2). En el volumen destinado a dormitorio podemos encontrar una plataforma elevada de unos 50 a 70 $\mathrm{cm}$ sobre el suelo en los lados angostos de la planta rectangular, denominada phatati. Está construida de adobe, de manera de conformar un cajón que deje espacio en su interior para almacenaje, el que se cierra con una tapa hecha de maderas, sobre esta se instala la cama propiamente tal, que se conoce como ikiña. Esta última está compuesta de varias pieles de llama y de frazadas tejidas de múltiples colores (Van Kessel 1996).

Las sucesivas situaciones de contacto con la cultura occidental (la colonización española, el Estado peruano y finalmente el Estado chileno) provocaron que progresivamente fueran apareciendo en el contexto de la vivienda tradicional un tipo de mobiliario externo, como sillas, mesas o camas de fierro o madera. Siendo la casa del pueblo central donde podemos ver primero la llegada del mobiliario occidental, por cuanto es aquí donde la influencia de la autoridad colonial y del Estado-Nación, posteriormente, se presentó con mayor fuerza.

Hoy las viviendas tradicionales o utas, aunque usadas de manera más esporádica, no parecen haber modificado de manera sustancial el modo de habitar particularmente respecto de la división de programas (cocina, dormitorio y almacenaje). Ha aparecido con mayor fuerza el mobiliario occidental, junto con artefactos e implementos como cocinas a gas o tambores plásticos y metálicos que han comenzado a ser utilizados para guardar agua o dentro del proceso de teñido de textiles. Sin embargo, en términos generales, las entrevistas y el trabajo en terreno comprobaron que el habitar en las zonas geográficas de las comunidades de origen sigue presentando ciertas características propias, que no parecen haber sido afectadas por la experiencia de habitación urbana.

\section{La vivienda urbana}

El explosivo aumento poblacional que vivió la ciudad de Arica implicó una importante ampliación de su espacio urbano, que se materializó principalmente mediante la ocupación espontánea (tomas de terreno, características de los años 1950-1960) o de 


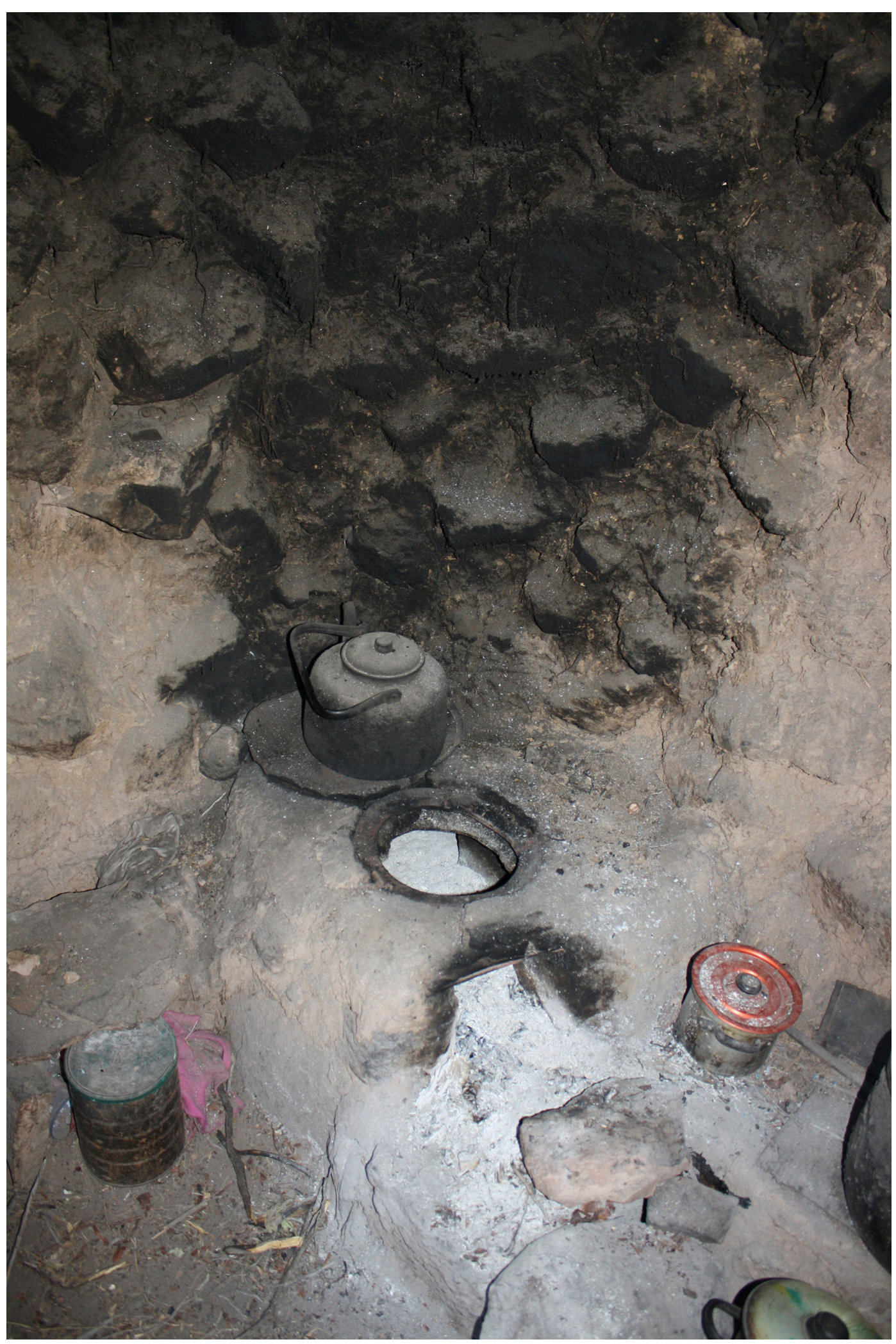

Figura 2. Vista interior de la cocina en una vivienda tradicional aymara en Itiza. 
medidas provistas por las mismas entidades gubernamentales para los sectores socioeconómicos más vulnerables (como la entrega planificada de sitios en las décadas 1960-1970 y de lotes con servicios durante 1970-1980). Desde fines de los años 1980 y particularmente los años 1990, la solución habitacional gubernamental se estructura a partir de la entrega de viviendas sociales, progresiva y básica (Ministerio de Vivienda y Urbanismo [MINVU] 2004), que limitan evidentemente la capacidad de agencia constructiva de los beneficiarios y privilegian la autoconstrucción desde un núcleo formal construido que contienen en sí mismo un modelo preestablecido de vivienda, con un programa de usos y recintos que no difiere a lo largo del país.

La construcción de viviendas sociales con espacios básicos definidos, aquellos de uso común y los de índole privado, generalmente en dos niveles, ha obligado a las familias aymaras migrantes a redefinir los espacios tradicionales presentes en las viviendas rurales en donde se desarrolla el habitar. Este desmantelamiento de la estructura tradicional, a pesar de parecer a primera vista radical, ha sido aminorado gracias a las mismas falencias que presentan las unidades básicas entregadas por el Estado. Normalmente, las familias beneficiarias de soluciones habitacionales comienzan un proceso de ampliación de las mismas por medio de la autoconstrucción. En el caso de la ciudad de Arica, esta realidad generalizada nos sirve para evidenciar cómo su instalación en la ciudad significa asumir nuevas condiciones de vida y habitar (González y Carrasco 2016).

Considerando que el programa propuesto por las viviendas sociales incluye un ideario programático que responde en su totalidad a conceptos occidentales respecto de cómo debe estar constituida una vivienda, podemos encontrar entonces una división de espacios que intenta ser nítida, separando un nivel como Living-Comedor-Cocina-Baño, aquellos pensados como de uso común y dos habitaciones en el segundo nivel que responden al ámbito familiar más privado. Este espacio inferior que se presenta como un recinto de uso común podríamos pensar se ajustaría para llevar a la vivienda en la ciudad el espacio central de la vivienda tradicional (ese espacio que hemos denominado como cocina, pero que integra otras actividades durante el día). Y sin embargo nos encontramos que en los casos de estudio la opción tomada por las familias aymaras ha sido la de marcar esa separación occidental programática, particularmente mediante una preocupación por articular un living o sala de estar por medio de la adquisición de un mobiliario acorde a la idea accidental de este espacio programático (Figura 3).

En términos históricos, el mobiliario ha estado implícitamente relacionado con la arquitectura como elementos que, en conjunción con el espacio construido y los objetos, completan el marco físico o material en el que se desarrolla el habitar. El diseño de muebles se puede considerar entonces, como un área en donde se puede leer la evolución histórica en el campo de la cultura material de los grupos sociales (Anderson 2015). Será a partir del siglo XIX cuando esta situación, si bien manifestada con anterioridad, adquiera mayor relevancia debido a que el diseño de muebles se vuelve más visible y con una carga teórica mayor, especialmente en naciones como Inglaterra gracias a la revolución industrial y la producción en serie, junto con movimientos como el Arts and Crafts, respuesta a este proceso industrial, que busca revitalizar la producción artesanal, cargando aún más de valor cultural a los muebles y el uso del mobiliario en general como símbolo material de cultura. Ya asentado el movimiento moderno, será en la Bauhaus donde se dará el puntapié para que el diseño industrial de mobiliario vinculado con el diseño académico se masifique, intensificando la relación entre el diseño de muebles desde un punto de vista histórico con los sistemas de estratificación social, idea planteada por Anthony Giddens a principios de la década de 1990 (Anderson 2015).

La sala de estar es un programa de uso de los espacios construidos que no existe, como hemos mencionado, en la vivienda tradicional aymara. Normalmente en los casos urbanos estudiados, este programa está compuesto por un set de sofás, uno para dos personas y dos simples, en la mayoría de los casos vinculado con el espacio de comedor, el que se compone también de un set de muebles (mesa rectangular para seis personas), siendo ambos grupos de mobiliarios claramente reconocibles como parte de los paquetes de oferta que son ampliamente ofrecidos por distintas tiendas comerciales. En aquellos casos estudiados que tienen mejor situación económica, observamos una mayor preocupación por el espacio de living + comedor, marcando claramente durante las entrevistas la importancia que posee este espacio como lugar de sociabilización con vecinos o amigos. Solamente en uno de los casos estudiados la familia 

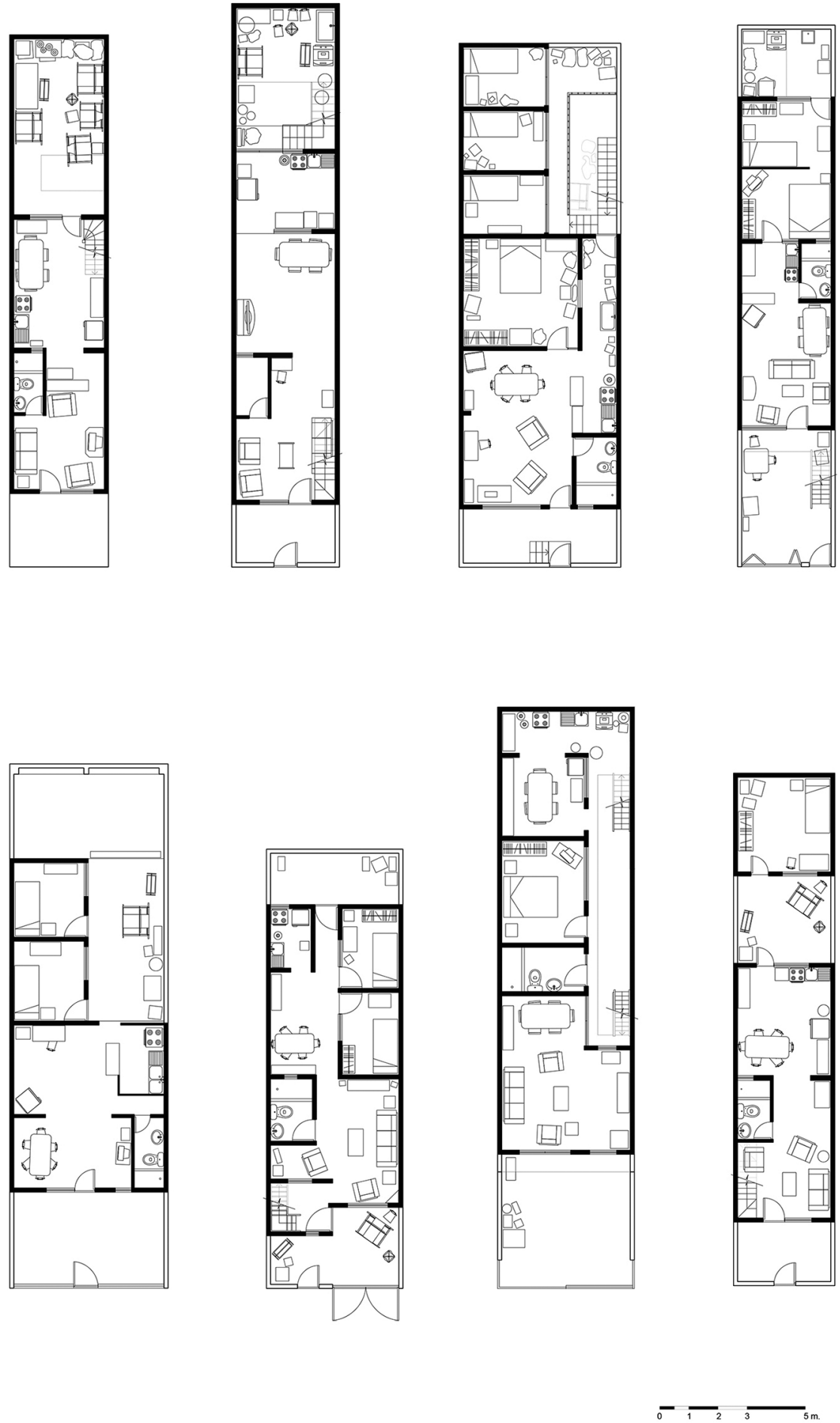

Figura 3. Ejemplo de las planimetrías de los casos de estudio de viviendas aymaras urbanas levantadas durante la investigación (casos 01-08). 
no contaba con una sala de estar, sin embargo era una preocupación relevante y que apareció tempranamente en la conversación, dejando claro que uno de los planes a futuro era el poder adquirir los muebles para generar este espacio. Es importante destacar que en ninguna de las viviendas levantadas planimétricamente pudimos registrar la presencia de algún tipo de elemento, adorno o amuleto que son comunes en la vivienda tradicional y que han sido descritos por autores que han documentado el simbolismo de la vivienda aymara (Palacios 1982; Arnold 1993).

Por su parte, la cocina (parte del programa de espacios comunes entregado en la unidad original), también presenta en su organización y provisión de mobiliario un avance de las ideas occidentales respecto de sus elementos y uso, pudiendo observarse en materia de artefactos en todos los casos el uso de cocinas a gas y refrigeradores. En materia de muebles podemos observar un mobiliario de cocina de tipo modular, que en mayor o menor medida intensifican la conformación de un modelo que se aleja del espacio tradicional descrito anteriormente. En dos viviendas de familias que contaban con una mejor situación económica pudimos observar cocinas completamente amobladas y que difícilmente se podrían diferenciar de la imagen ideal del espacio que se publicita comercialmente en la totalidad del país.

Ante la consulta realizada en el proceso de entrevistas, acerca de cuál es el espacio más importante de la vivienda en la ciudad, fue el living o sala de estar, junto con la cocina, los que aparecieron como los espacios más mencionados. Para la mayoría de la familia la sala de estar, como espacio definido inequívocamente en conjunción con el mobiliario que la compone, fue descrita como lugar de presentación y sociabilización de la familia con el resto de la comunidad (amigos, familia y vecinos), mencionado especialmente respecto de familias con miembros jóvenes aún en etapa de estudio (primario, secundario y universitario) como lugar de recepción de los compañeros de sus hijos. Por su parte, la cocina apareció mencionada en un sentido más próximo al que tendría en la vivienda tradicional, como el lugar de reunión más privado. Fue descrito como un espacio vedado a los visitantes ajenos al núcleo familiar, reforzando la idea de que es la sala de estar el espacio que sirve de instancia construida de relación con personas exteriores de la familia.

\section{Conclusiones}

Si bien la ciudad pudo haber sido pensada como un espacio en donde la diversidad cultural y étnica, especialmente en el caso de las ciudades portuarias como Arica e Iquique, no hubiesen debido acarrear diferencias significativas respecto de ser o no aymara, tempranamente la sociedad regional y urbana terminó por evidenciar una mirada discriminante. La ciudad se transformó entonces en un nuevo escenario de marginación, por cuanto durante el proceso de instalación en ella las familias migrantes aymaras fueron relegadas a ciertos barrios e ingresaron con la categoría de pobladores marginales (González y Gavilán 1990) dentro del macrosegmento de la sociedad urbana catalogada como de bajos ingresos.

La condición de enajenación social definitiva que se produce inicialmente en el arribo e instalación en la ciudad terminó ocasionando que durante las primeras décadas de residencia en las urbes, la posibilidad de ascenso social por medio de la acumulación de bienes o de la educación fuesen marginales y la única opción posible de integración a esta sociedad urbana se diera por medio del "blanqueamiento" (González y Gavilán 1990). Este se manifiesta en distintas esferas de significación y acción, pero que seguramente tiene en el ámbito del aspecto físico propiamente tal, su más significativa manifestación, pudiéndose observar el uso de maquillaje exagerado o el teñido del cabello o durante las primeras décadas buscando parejas que fueran "blancos", de manera de provocar un mestizaje que les permitiera una mejor aceptación respecto de la población no indígena predominante en las ciudades del norte de Chile, especialmente en Arica (Chipana 1986).

Creemos que la aparición y aceptación de nuevos espacios del habitar doméstico en la vivienda aymara urbana, se puede leer dentro de esta misma línea de acciones tomadas por las familias migrantes para lograr encajar dentro de los códigos de habitación propios de la sociedad chilena, marcadamente occidentalizada en la espacialización de sus viviendas. La creación formal de estos espacios y el uso de mobiliario registrada en las planimetrías levantadas de las viviendas estudiadas y el material extraído de las entrevistas, refuerza la noción de que el espacio de living, aquel que es entendido como la carta de presentación de la vivienda, busca hacer desaparecer una barrera física, material y cultural 
que haga latente las diferencias que existen entre las costumbre en el habitar tradicional, aquel que sin embargo es claramente descrito por las familias, y la realidad homogénea de la urbe. De la misma manera que las personas de esta etnia cambiaron su manera de vestir o el color de su cabello como describe Chipana (1996), la casa urbana fue objeto también de un proceso de cambio para parecerse a esa mayoría homogénea que los miraba como un otro.

Desde el término de la dictadura militar, los esfuerzos de los sucesivos gobiernos democráticos por exponer el problema indígena como una variable importante terminó con la valorización positiva del concepto de diversidad cultural nacional, ha permitido que desde los esfuerzos gubernamentales la mantención de la identidad indígena y los valores y beneficios agregados que esta pueda acarrear (particularmente fondos y becas dirigidas al segmento indígena) haya ocasionado un crecimiento sostenido de aquellos que se designan como aymaras en la región y, por consiguiente, el posicionamiento de este segmento de la ciudad urbana en el total ha crecido hasta formar parte prácticamente de las características propias y destacadas de la ciudad. Ha surgido una identidad aymara entre la población indígena del norte grande de Chile; principalmente los aymaras urbanos sienten la necesidad de articular una memoria representativa de ese colectivo amplio que es el pueblo aymara (Zapata 2007). Esta nueva identidad deberá obligatoriamente comenzar a ser visibilizada por los entes estatales encargados de la planificación de la ciudad y de las viviendas entregadas por el Estado. Así, el análisis de las características de las viviendas aymaras urbanas es fundamental para llegar a una solución que integre las necesidades culturales de este grupo étnico, ya no respecto de la situación tradicional, sino que de una realidad ya existente en la que cohabitan costumbres tradicionales y adaptaciones no menores en el habitar urbano y su espacio construido.

\section{Agradecimientos}

Quisiera agradecer al programa CONICYT Becas Chile, Doctorado en el Extranjero.

\section{Referencias Citadas}

Aceituno, $\mathrm{P}$.

1996 Elementos del clima en el sudamericano. Revista Geofísica del Instituto Panamericano de Geografía e Historia, Enero-Junio. pp. 37-55.

Anderson, I. F.

2015 Teoría y crítica del diseño de muebles. Revista Arte e Investigación, año 17 (11), pp. 20-26.

Arnold D. et al.

1993 Hacia un orden andino de las cosas, Hisbol/ICA, La Paz.

Canals, F.

1959 Las civilizaciones prehispánicas de América. Editorial Sudamericana, Buenos Aires.

Chipana, C.

1986 La identidad étnica de los aymaras en Arica. Chungara Revista Chilena de Antropología, vol. 16-17. pp. 251-261.

González, D. y Carrasco A. M.

2016 El patio, espacio mediador. Características del habitar tradicional aymara presentes en viviendas sociales de familias residentes en Arica, Chile. Interciencia, febrero Volumen 41 $\mathrm{N}^{\circ}$ 2. pp. 92-97.

González, H. y V. Gavilán

1990 Cultura e identidad étnica entre los aymaras chilenos. Chungara Revista Chilena de Antropología, vol. 24-25. pp. $145-158$.

González, H. Gundermann, H. e Hidalgo, J.

2014 Comunidad indígena y construcción histórica del espacio entre los aymara del norte de Chile. Chungara Revista Chilena de Antropología, vol. 46, N² 2. pp. 233-246.
González, S.

2004 El Dios Cautivo. Las Ligas Patrióticas en la chilenización compulsiva de Tarapacá(1910-1922), LOM Ediciones, Santiago.

INE.

2002 Estadísticas Sociales de los Pueblos Indígenas en Chile, Censo 2002. Instituto Nacional de Estadísticas, Santiago. INE.

2003 Censo de población y vivienda. Resultados. Instituto Nacional de Estadísticas, Santiago.

MINVU

2004 Chile, un siglo de politicas en vivienda y barrio, Pehuen Editores, Santiago.

Muñoz, I.

2012 Hurgando la vivienda andina a través de la historia: percepción y ocupación del espacio domésticoceremonial en los valles y altiplano en la región de Arica y Parinacota, Chile. Intersecciones en Antropología 15. pp. 235-250.

Palacios F.

1982 El simbolismo aymara de la casa. Boletín del Instituto de Estudios Aymaras, Vol. 2 No 12. pp. 37-57.

Solc, V.

1975 Casa Aymara en Enquelga. Annals of the Náprstek Museum $\mathrm{N}^{\mathrm{o}} 8$, pp. 111-146.

Tudela, P.

1993 Chilenización y cambio ideológico entre los aymaras de Arica (1883-1930). Intervención religiosa y secularización. Revista Chilena de Antropología $\mathrm{N}^{\circ}$ 12. pp. 201-23. 


\section{Van Kessel J.}

1996 Los aymaras contemporáneos en Chile, en Etnografía. Sociedades Indígenas Contemporáneas y su Ideología, editado por J. Hidalgo et al., pp. 46-67, Editorial Andrés Bello, Santiago.

\section{Zapata, C.}

2007 Memoria e historia. El proyecto de una identidad colectiva entre los aymaras de Chile. Chungara Revista Chilena de Antropología, vol. 39 num. 2. pp. 171-183. 